\title{
Characterization of Facade Regularities in High-Resolution SAR Images
}

\author{
Stefan Auer, Christoph Gisinger, and Junyi Tao
}

\begin{abstract}
The grammar of facade structures is often related to regularly distributed signature patterns in high-resolution synthetic aperture radar (SAR) images. Given those patterns in the imagery, they should be used as a source of information for identifying changes related to the facade. This paper presents a method for characterizing the layover area pertinent to regularly arranged facade structures, formulated on a general basis for single azimuth/range SAR images and geocoded SAR images. The analysis follows assumptions on the intensity distribution, the linear arrangement, and the regularity of point-like signatures. Two case studies on facades are presented, which confirm the applicability of the method for different building types. Based on that, the potentials and limitations of the algorithm are discussed with respect to applications such as change detection and persistent scatterer interferometry.
\end{abstract}

Index Terms-Grouping, high-resolution synthetic aperture radar (SAR), pattern recognition, simulation, spectral analysis, TerraSAR-X, urban.

\section{INTRODUCTION AND MOTIVATION}

$\mathbf{S}$ YNTHETIC aperture radar (SAR) sensors, either airborne [1], [2] or spaceborne [3], [4], with meter- or submeter resolution, show high potential for the monitoring of urban areas. In this context, structural elements of buildings such as windows, balconies, or roof details, mostly composed of canonical shapes such as dihedrals, trihedrals, or cylinders, often correspond to dominant signatures in the SAR images. A review of the state-of-the-art reveals the relevance of those signatures for the extraction and monitoring of individual buildings. For instance, methods for interferometric processing such as persistent scatterer interferometry (PSI) [5] or SAR tomography (TomoSAR) [6] analyze temporal coherent pixels (point targets) in order to estimate the 3-D position and deformation of such urban structures. In the field of building reconstruction, shape parameters are determined based on salient SAR image features, e.g., from single SAR images [7], multiaspect SAR

Manuscript received December 18, 2013; revised June 13, 2014 and September 23, 2014; accepted October 13, 2014. This work was supported in part by Deutsche Forschungsgemeinschaft (DFG), project VHR-SAR (BA 2033/3-1).

S. Auer is with the Chair of Remote Sensing Technology, Technische Universität München (TUM), 80333 München, Germany (e-mail: S.Auer@tum.de).

C. Gisinger is with the Institute for Astronomical and Physical Geodesy, Technische Universität München (TUM), 80333 München, Germany (e-mail: Christoph.Gisinger@bv.tum.de).

J. Tao is with the Remote Sensing Technology Institute (IMF), German Aerospace Center (DLR), 82234 Oberpfaffenhofen, Germany (e-mail: Junyi. Tao@dlr.de).

Color versions of one or more of the figures in this paper are available online at http://ieeexplore.ieee.org.

Digital Object Identifier 10.1109/TGRS.2014.2364076

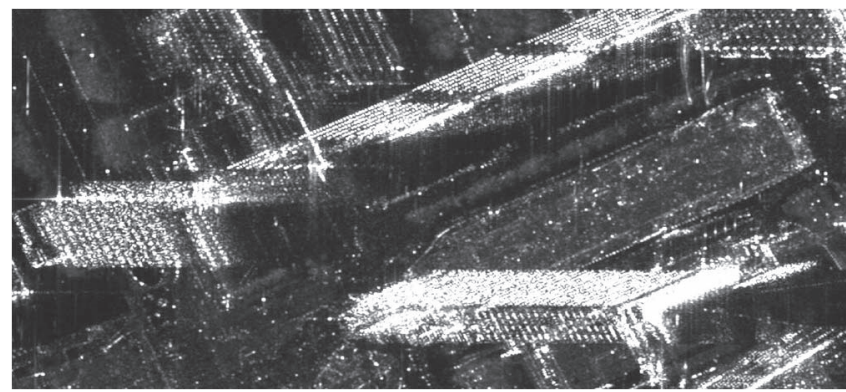

Fig. 1. Signature patterns related to buildings in Berlin city center; clipped average image of a data stack captured in TerraSAR-X high-resolution spotlight mode. Spatial resolution: $1.1 \mathrm{~m}$ in azimuth and $0.6 \mathrm{~m}$ in range. Range increasing from left to right.

data [8], or from images captured with different incidence angles [9].

Fig. 1 shows an urban scene of the city center of Berlin, Germany, imaged by TerraSAR-X in high-resolution spotlight mode (see [10] for details on the sensor). The image has been generated by the temporal averaging of a SAR image stack. Thus, deterministic signal contributions from buildings are prominent, whereas random signal noise is suppressed. From long-term experience with TerraSAR-X high-resolution data, it is known that approximately 50\% of the long-time coherent scatterers in urban areas are related to facades [11]. In this context, facades are often represented by regular signature patterns. As an example, pattern regularities are distinguishable in Fig. 1. The patterns strongly vary in intensity due to differences of the size and the material of the facade structures. Given the structural details in the SAR image, algorithms for building extraction/monitoring should exploit this contextual information for an object-based analysis.

Following this idea, this paper is focused on extracting regularities in facade layover areas based on salient point signatures. The work is driven by the aim of supporting interferometric methods (PSI and TomoSAR), change detection, and object reconstruction in future applications. Related work is mainly reported in the context of building extraction and PSI. In Simonetto et al. [9], linear features and line intersections are used for stereoscopic building reconstruction, including a local Hough analysis. In GESTALT, a production system for building extraction, extracted lines, and groups of point signatures are utilized as building features [12], [13]. In the field of PSI, the positions of long-time coherent persistent scatterers (PS) are analyzed for regularities. Algorithms for grouping PS are proposed by Schunert et al. [14] using GESTALT and Schack et al. [15] in order to improve the 3-D localization capability of PSI, the latter including optical data with oblique perspectives. 
This article has been accepted for inclusion in a future issue of this journal. Content is final as presented, with the exception of pagination.

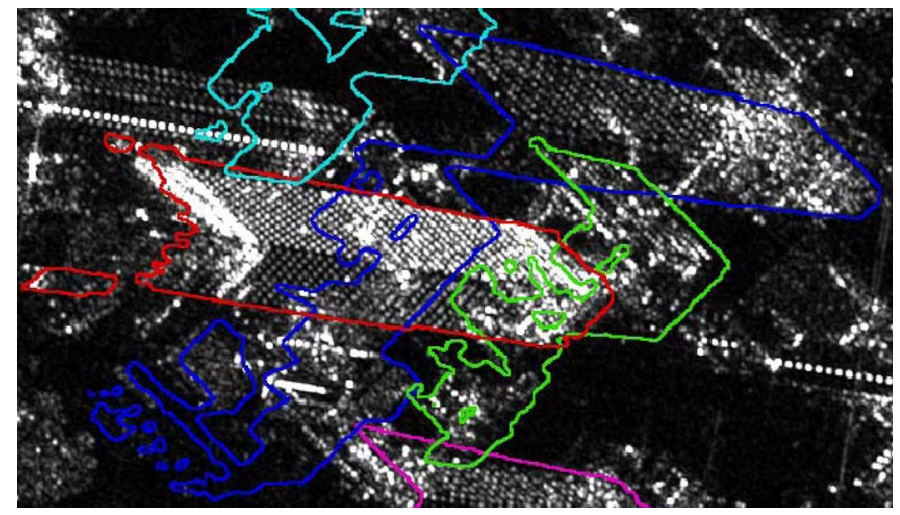

Fig. 2. Simulated extent of layover areas for an urban scene in San Francisco. Facade signatures of different buildings are partly superimposed. The red outline marks the skyscraper analyzed in Section IV-B. East: left-to-right. North: bottom-up.

The work presented in this paper is focused on extracting point signature patterns of facades from single SAR images, given the layover extent and orientation of individual facades. In this context, the pattern recognition concept is based on knowledge from simulation case studies indicating the correspondence between point signatures and facade structures [4], [16], [17]. The proposed methodology for pattern extraction addresses three new aspects.

1) A new method for extracting patterns from facade layovers is introduced, integrating four components: radiometric analysis, line fitting, spectral analysis, and directed grouping.

2) The spatial distribution of prominent point-like signatures is considered as the main source of information in the layover area.

3) The algorithm is formulated on a general basis, supporting the analysis of SAR images in azimuth/range (e.g., PSI) and geocoded images (e.g., change detection).

The remainder of this paper is structured as follows. Basics, prerequisites, and an overview of the concept for pattern extraction are presented in Section II. Thereafter, the components of the algorithm are introduced in Section III. Two case studies are presented in Section IV, demonstrating the potentials and limitations of the approach. The conclusion and an outlook to future work are given in Section V.

\section{REQUIREMENTS AND CONCEPT}

\section{A. Integration of Object Knowledge}

The algorithm for pattern characterization is based on the known facade layover extent and an approximate orientation angle. Concepts for extracting this knowledge based on SAR data are, for instance, reported for single SAR images [7] and for interferometric data [18]. In this paper, the layover outline is determined using a simulation processing chain including RaySAR [19], [20].

A digital surface model (DSM), generated from LiDAR data, is decomposed into facade models based on height and gradient information in order to simulate the layover areas of individual facades [21]. As an example, Fig. 2 indicates the potential for an urban scene in San Francisco. The simulation of geocoded
SAR images of the building models enables the ability to define and extract the corresponding layover areas in the TerraSAR-X image (layover outlines marked by colors). Note that the pattern recognition concept proposed in this paper is focused on individual facades. Correspondingly, the simulation step is conducted for models of individual facades instead of complete buildings.

The pattern recognition algorithm is based on the layover pixels and the layover orientation of individual facades which are provided in the course of the simulation procedure. The orientation angle of the facade surface, derived during the extraction of facade models from the DSM, is required for steering the search for regularities in the layover area. The geocoded layover pixels of facades are provided in rectangular frames where nonlayover pixels are set to 0 [see example in Fig. 9(a)]. Given the area of interest, the subsequent pattern extraction is based on the expectation of salient signatures corresponding to facade elements.

Summarized, the integration of simulation and pattern recognition can be described as follows. The simulation provides the area of interest (layover pixels) and the facade orientation angle from the DSM. Thereafter, the recognition step extracts the arrangement of signatures in the area of interest as source of information on geometric structures on the facade.

\section{B. Concept of Pattern Extraction}

The main motivation of the work presented in this paper was to develop a method which works independently from the facade appearance (intensity and signature distribution) and the number of patterns related to a facade. Focused on single SAR images, the algorithm has to deal with strong signature variations related to the variation of facade geometries and materials. Subsequent processing steps are used to separate pattern candidates from isolated point signatures and to extract the actual pattern. First, pattern candidates are selected based on intensity criteria (Section III-A). Thereafter, candidates are discarded based on line fitting (Section III-B), and dominating spacing values are extracted (Section III-C). Finally, a local grouping procedure defines the topological order of the remaining pattern members (Section III-D).

Several assumptions underlie the algorithm. First, structures of equal type on a facade are expected to yield similar intensities in the layover image. Moreover, regular arrangements of facade structures are expected to be related to signature regularities. Finally, one facade may be represented by several subpatterns with equal or different spacing. Structures on roofs may also contribute to regularities of the layover area. Therefore, the analysis is focused on 2-D patterns as indicators for the 2-D distribution of structures on facades (window corners, balconies, etc.).

\section{Methodology}

Based on the orbit meta file of the TerraSAR-X image and the simulation step, the algorithm integrates the following input information.

1) A rectangular SAR image section $u_{1}(x, r)$ (facade layover) of height $h$ and width $w$, where nonlayover pixels 
TABLE I

INPUT PARAMETERS For PROCESSING STEPS. BOLD: MANUALly Defined Parameters. Other Parameters ARe BASED on SAR DATA/Building MOdel OR DeRIVEd Automatically During Processing STEPS (Note: Parameters $\Delta_{\theta r}$ AND $\Delta_{\theta s}$ Are Manually Selected in This Paper But MaY ReLY on THE INPUT Data AS Well)

\begin{tabular}{|l|l|}
\hline $\begin{array}{l}\text { Line fitting } \\
\text { (section III-B) }\end{array}$ & $\mathbf{d}_{\mathbf{i}}, \mathbf{f}_{\mathbf{H}}$ \\
& $\begin{array}{l}\theta_{f}, \theta_{r}, \Delta_{\theta s}, \Delta_{\theta r}, \\
\delta_{x}, \delta_{r}, \Delta x, \Delta r \\
h, w\end{array}$ \\
\hline $\begin{array}{l}\text { Spectral analysis } \\
\text { (section III-C) }\end{array}$ & $\mathbf{d}_{\mathbf{s}}$ \\
& $\begin{array}{l}\theta_{f}, \theta_{r}, \Delta_{\theta s}, \Delta_{\theta r}, \\
\Delta x, \Delta r\end{array}$ \\
& $h, w$
\end{tabular}

are set to 0 . The parameters $x$ and $r$ represent either axes in azimuth/range or north/east direction (geocoded data).

2) The image rotation angle $\theta_{r}$ related to geocoding with an uncertainty parameter $\Delta_{\theta r}$ [degrees] $\left(\theta_{r}=0^{\circ}\right.$ for azimuth/range geometry). Both parameters may stem from the geocoding procedure.

3) The shearing angle $\theta_{s}$ of the facade with respect to the line-of-flight of the sensor with an uncertainty parameter $\Delta_{\theta s}$ [degrees], both possibly relying on the method for layover extraction. The sum of $\theta_{s}$ and $\theta_{r}$ is equal to the facade orientation $\theta_{f}$ in the SAR image.

4) The spatial resolution of the SAR image in azimuth $\delta_{x}$ and range $\delta_{r}$ as well as the pixel spacing $\Delta x$ and $\Delta r$ in azimuth/range or north/east [meters] (depending on the image geometry).

The rotation angles $\theta_{r}$ and $\theta_{s}$ are defined with respect to the bottom-up axis of the image (positive for clockwise rotation). In addition, the pattern analysis is initiated with the following parameters:

1) The minimum number of signature rows $\min _{r}$ and columns $\min _{c}$ to be accepted as a pattern.

2) Two distance thresholds for fitting point signatures to linear arrangements (threshold $d_{i}$ ) and regions (threshold $\left.d_{r}\right)$ [subpixels].

3) A truncation factor $f_{H}$ for defining a threshold for line detection [interval: $0-1$ ].

4) A maximum spacing value $d_{s}$ (meters) is required for discarding (dominant) low-frequency parts in the layover spectrum. For the case studies presented in this paper, $d_{s}$ is set to $10 \mathrm{~m}$.

In Section IV, a discussion of the impact and relevance of the parameters is given in the course of two case studies referred to as "TUM Nordbau" and "Skyscraper." Table I gives an overview of all parameters considered by the processing steps.

\section{A. Selection of Candidates}

The peak position of point targets (corner reflectors) is located in one resolution cell [22]. Accordingly, the search of appropriate signatures, referred to as pattern candidates in the following, is started by extracting local maxima in the layover area. For each candidate, the peak position is locally refined to subpixels within a window of $3 \times 3$ pixels, which is oversampled by a factor of 32 . Based on that, the subsequent spatial assignment of pattern candidates to lines and patterns is conducted with subpixel precision. Thereafter, the set of pattern candidates is reduced based on the intensity distribution. First, candidates of low intensity are discarded by means of a threshold (median intensity in layover area). Second, isolated prominent point signatures are removed following the assumption that regularly distributed targets on the same facade are made of similar material leading to similar intensities. The distribution of intensities is assumed to follow a log-normal distribution, which serves as a rough representative for urban areas in high-resolution data [23]. After calculating the mean value and the standard deviation of the logarithmically scaled intensities, dominant outliers are discarded by a confidence interval of $95 \%$. Two examples for the extraction of pattern candidates are given in Section IV. Therein, dominant isolated signatures are discarded, whereas signatures related to the patterns are preserved.

\section{B. Detection of Linear Arrangements}

The next step of the algorithm identifies lines within the layover by exploiting the linear arrangement of the pattern candidates. Thereafter, isolated pattern candidates not fitting to the lines are discarded. The detection of line features is based on an orientation-bounded Hough transform [24], [25], where maxima indicate the presence of lines (Hough space axes: perpendicular distance $n$ to the coordinate origin with a spacing of 1 pixel, angle $\theta$ with respect to the horizontal image axis with a spacing of $1^{\circ}$ ). As a prerequisite, a binary image is generated marking the pixel-positions of pattern candidates in the layover area.

Additional information can be introduced to reduce the workload and to improve the robustness. In general, the complete set of angles $-90^{\circ} \leq \theta \leq 89^{\circ}$ has to be tested to generate the Hough space for line detection. However, the $\theta$ angle is approximately known from the layover orientation angle (cf., Section II-A). Along this direction, facade structures (e.g., windows and wall corners) are expected to be aligned on different floor levels. In case of a geocoded image, the angle further includes the rotation angle $\theta_{r}$. Consequently, the angles for the Hough transform can be reduced to a small band which includes lines pointing along the facade $\left(\theta_{f} \pm \Delta_{\theta s}\right)$.

Fig. 3 shows the Hough space for a facade layover imaged by TerraSAR-X, where the search interval is marked. The limitation to the interval is mandatory as there are many options for fitting lines to a regular grid of point signatures, leading to additional peaks throughout the Hough space.

In the interval, entries succeeding a threshold (Hough space maximum scaled by factor $f_{H}$ ) are extracted, which define a 


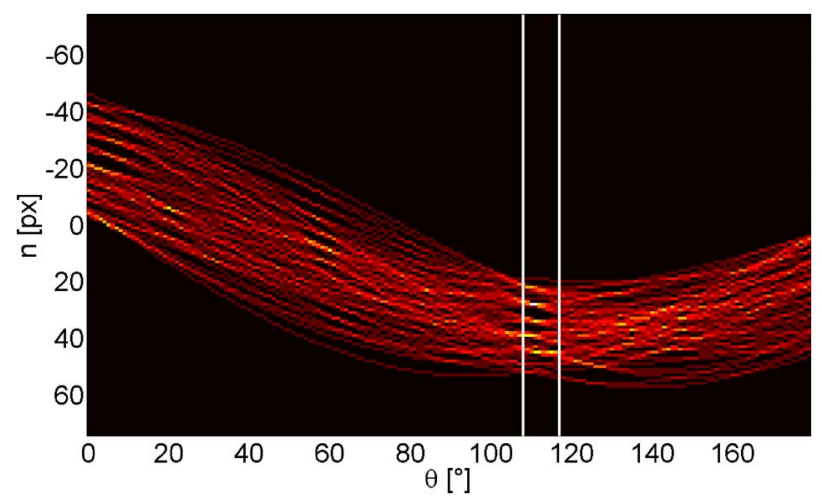

Fig. 3. Hough space for facade "TUM Nordbau" (incidence angle: $50.0^{\circ}$ ). The extraction of line candidates is restricted to an interval (marked by white lines) based on a priori knowledge of facade orientation (cf., Section IV-A for details on the case study).

set of potential lines. In this context, the maximum number of line candidates to be extracted is $n_{1}=\|w / 2\|$ along the facade (i.e., corresponding to structures on the same floor level), $\|\cdot\|$ rounding to the next integer, where $w$ is the width of the layover image section (unit: pixel). We can assume a certain parallelism for lines making up the facade pattern. Since the search window was already narrowed down by specifying a reduced Hough space, the median $\theta$ value of the remaining line candidates combined with a buffer of $1^{\circ}$ assesses the degree of parallelism, and thereby, the amount of candidates is further reduced. Thereafter, the distance component $n$ is investigated. Considering the properties of the layover image (cf., Section II-A), the minimum spacing between two lines is set to $\Delta \rho_{1}=\left\|\delta_{x} / \Delta x\right\|$ along the facade direction. The minimum spacing is used to group the remaining line candidates to bundles from which the lines with the largest Hough space values are extracted.

Based on the detected lines along the facade, the number of pattern candidates is reduced. Candidates whose spatial distance to the nearest line is higher than the criterion $d_{i}$ are discarded. Note that the criterion is not supposed to be rigorous as the focus is on the removal of clear outliers.

The known positions of the remaining pattern candidates are used for creating a new layover image. Local windows (size: $3 \times 3$ pixels) are extracted at the candidate positions and weighed with a binomial filter in order to dampen high frequencies at the window margins. Thereafter, the local windows are inserted to an empty image layer-finally giving $u_{2}(x, r)$ - which is the input to the subsequent spectral analysis based on a Fourier transform. Compared to the original layover image $u_{1}(x, r)$, dominant frequencies are more prominent in $u_{2}(x, r)$, supporting the extraction of spacing and orientation values in the spectrum. Fig. 4 shows an example for a layover image of a facade. The emphasis of dominant frequencies is crucial in case of a high number of isolated signatures disturbing the layover pattern(s).

\section{Spectral Analysis}

Regular distributions of signatures in images correspond to dominant frequencies distinguishable in the spectrum which, for instance, can be exploited in the context of texture analysis and synthesis [26]-[28]. In case of the preprocessed SAR
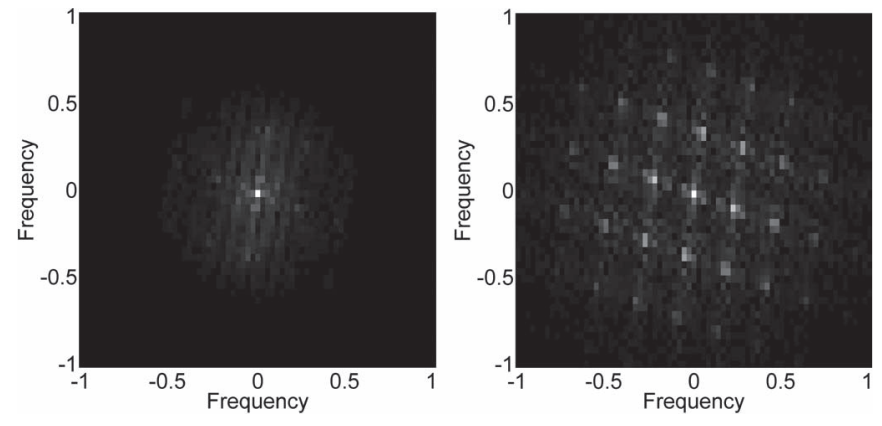

Fig. 4. Enhancement of spectral signal for facade "Nordbau" (incidence angle: $39.1^{\circ}$ ) by removing pattern candidates not fitting to the Hough lines. (Left) Spectrum related to $u_{1}(x, r)$. (Right) Spectrum related to $u_{2}(x, r)$. The maxima 1 of the (normalized) frequencies correspond to a spatial distance of 1 pixel in azimuth and range, respectively. See Section IV-A for details on the case study.

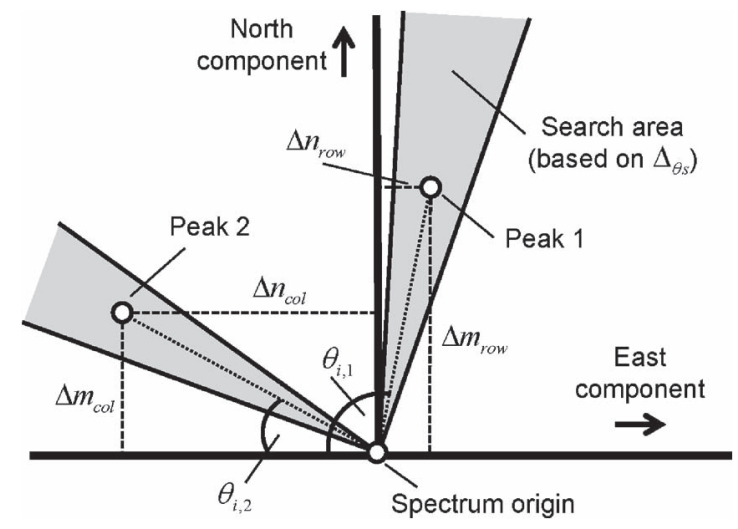

Fig. 5. Extraction of dominant peaks in the layover spectrum. Peak 1 contains information about the spacing along the facade and the geocoding rotation angle. Peak 2 contains information about the spacing in range and the facade orientation angle. Depending on the facade orientation, peak 2 appears to the left or to the right of the spectrum origin.

layover image $u_{2}(x, r)$, spectral peaks represent the dominant spacing values between pattern candidates as well as the facade orientation angle. The analysis is supported by a priori knowledge about the layover image.

Only the upper half of the spectrum is required for the subsequent identification of dominant peaks (cf., Fig. 5). The rotation of the range axis during geocoding relates to a rotation and scaling of the along-facade component in the spectrum. The facade rotation with respect to the sensor's line-of-flight corresponds to the rotation and scaling of the range component in the spectrum. Accordingly, the interdependence of the azimuth/ range and north/east axes has to be taken into account for obtaining appropriate spacing values for the subsequent grouping procedure.

Given at the start, approximate angles of interest are known, i.e., $\theta_{i, 1}=\theta_{r}+90^{\circ}$ for the facade orientation and $\theta_{i, 2}=\theta_{r}+$ $\theta_{s}$ for the range direction. Dominant peaks, likely representing the regularity of facade structures, are expected along those directions. Hence, spectrum entries of interest for finding regularities are restricted to two angle intervals (see Fig. 5; limits: $\theta_{i, 1} \pm 3 \cdot \Delta_{\theta s}$ and $\theta_{i, 2}+3 \cdot \Delta_{\theta r}$ ).

Only spectrum entries in between the resulting two circle segments are preserved for the subsequent analysis, whereas 


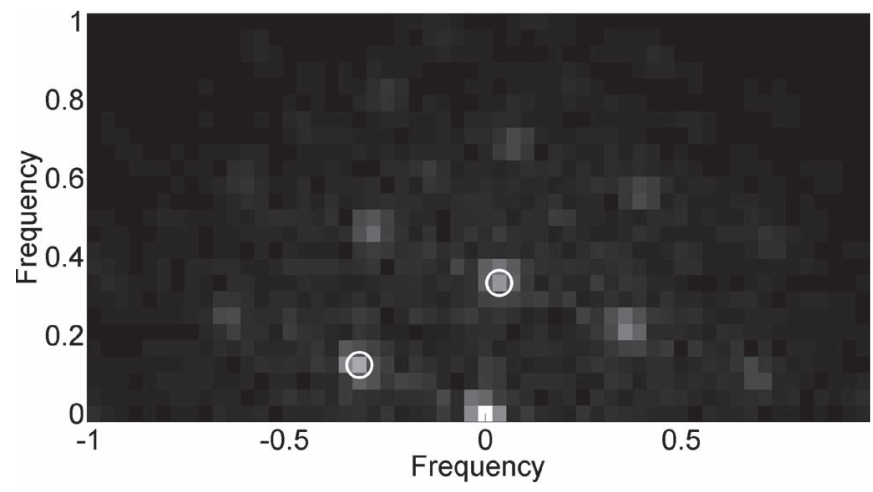

Fig. 6. Peaks (white circles) extracted in spectrum of layover "TUM Nordbau" (incidence angle: $39.1^{\circ}$ ) for identifying dominant spacing values and the shearing angle of the pattern [with respect to the spectrum origin with coordinates $(0,0)]$. See Section IV-A for details on the case study.

other spectrum parts are set to 0 . Pixels near the spectrum origin are removed as well, as they only represent the average intensity in the layover area. The frequency threshold in both directions is $f_{\min }=1 / d_{s}$, where $d_{s}$ is the maximum spacing value to be detected. Thereby, the spectrum is restricted to two search areas, which is comparable to a Gabor filter (e.g., [29]) with two orientations.

The position of the spectrum maximum is extracted within each angle interval. In case of two or more maxima of equal strength in the same angle interval, the peak with the lowest frequency is favored. As shown in Fig. 5, peak 1 primarily contains information about the spacing along the facade, whereas peak 2 contains information about the spacing in range and the facade orientation angle. As an example, Fig. 6 shows the extracted peaks for a facade layover imaged by TerraSAR-X.

Using the coordinates of the peaks, the spacing values along the axes are calculated with respect to the spectrum origin, resulting in (spectral) pixel distances along the facade $\Delta m_{\text {row }}$, $\Delta n_{\text {row }}$ and in range $\Delta m_{\mathrm{col}}, \Delta n_{\mathrm{col}}$, related to the rows and columns of the pattern. Thereafter, the orientation angle, including the rotation inherent to geocoding, is derived by

$$
\theta_{f}=\arctan \left(\frac{\Delta m_{\mathrm{col}} \cdot \Delta f_{m}}{\Delta n_{\mathrm{col}} \cdot \Delta f_{n}}\right)
$$

and wrapped to the interval $-\pi$ to $\pi$ with $\Delta f_{m}$ and $\Delta f_{n}$ being the frequency step widths in the spectrum. The dominant frequency along the facade is

$$
\Delta f_{\text {row }}=\sqrt{\left(\Delta m_{\text {row }} \Delta f_{m}\right)^{2}+\left(\Delta n_{\text {row }} \Delta f_{n}\right)^{2} \cos \left(\left|\theta_{f}-\theta_{r}\right|\right)}
$$

considering the rotation angle of the facade with respect to the sensor's line-of-flight. The corresponding spacing value $d_{\text {row }}$ along the facade in the SAR image is decomposed into distances in the SAR image

$$
\begin{aligned}
& \Delta x_{\text {row }}=d_{\text {row }} \cdot \cos \left(\theta_{f}\right) \\
& \Delta r_{\text {row }}=-d_{\text {row }} \cdot \sin \left(\theta_{f}\right) .
\end{aligned}
$$

In this context, $\Delta x_{\text {row }}$ and $\Delta r_{\text {row }}$ represent step widths topdown and left-right in the image, corresponding to counter- azimuth and in-range or to south and east (geocoded SAR image). The dominant frequency component in range is

$$
\Delta f_{\mathrm{col}}=\left|\Delta n_{\mathrm{col}} \cdot \Delta f_{n}\right|-\left|\Delta m_{\mathrm{col}} \cdot \Delta f_{m} \cdot \tan \left(\theta_{r}\right)\right|
$$

which corresponds to the spatial distance $d_{\text {col }}$ and two components along the SAR image axes

$$
\begin{aligned}
& \Delta x_{\mathrm{col}}=d_{\mathrm{col}} \cdot \cos \left(\theta_{r}\right) \\
& \Delta r_{\mathrm{col}}=d_{\mathrm{col}} \cdot \sin \left(\theta_{r}\right) .
\end{aligned}
$$

Finally, the spatial distances $\Delta x_{\text {row }}, \Delta r_{\text {row }}, \Delta x_{\text {col }}$, and $\Delta r_{\text {col }}$ are transformed to subpixel step widths based on the known pixel spacing of the SAR image.

\section{Directed Grouping}

The aim of the grouping procedure is to identify patterns which fit to the dominant spacing extracted from the layover spectrum. No assumption is made on the number of patterns in the layover area. Thereby, the extraction of spatially separated subpatterns with the same spacing is possible. Inherently, the grouping includes a test whether nearby pattern members are spatially connected. Hence, isolated signatures in the pattern are avoided.

For initiating the process, the subpixel positions of pattern candidates fulfilling the Hough line criterion (cf., Section III-B) are used as seed points. First, the search is conducted along the range direction as long as appropriate signatures are identified. Thereafter, the algorithm aims at adding further rows along the facade, where the search for pattern members is continued in range direction, respectively. Candidates are searched for in a circle with radius $d_{r}$ centered at the expected pattern position. In case several candidates are present, the decision is made based on the smallest spatial difference. The search for a new member is always started from the subpixel position of a pattern member.

Fig. 7 shows the grouping procedure. Starting with the topleft signature in the layover area as a seed point, signatures are collected within the first row (black arrows). Each signature is remembered as a possible seed point for the move to row 2 . At each signature position, upward moves are possible (indicated by gray arrows). If no further hit is identified in row 1 , the group of pattern signatures is expanded in the next row by a search to the left and right. Pattern candidates missing in rows 3 and 4 are bypassed by a move from a back-up seed point in row 3 . Starting from row 5, the gap within the pattern is surrounded by moves upward as long as further pattern members are identified. Each signature fitting to the pattern is assigned with a local pattern coordinate, starting with the first seed point $[1,1]$. Finally, the combination of signature IDs and pattern coordinates is parsed to a topology matrix with signature IDs as entries, defining the neighborhood of the pattern members (gaps marked by zeros). As an example, the pattern in Fig. 7 results in a $6 \times 6$ topology matrix with 4 zeros.

The grouping concept may be affected by orientation errors in case of long bypasses in the pattern due to gaps. This may result in a localization problem causing erroneous row 


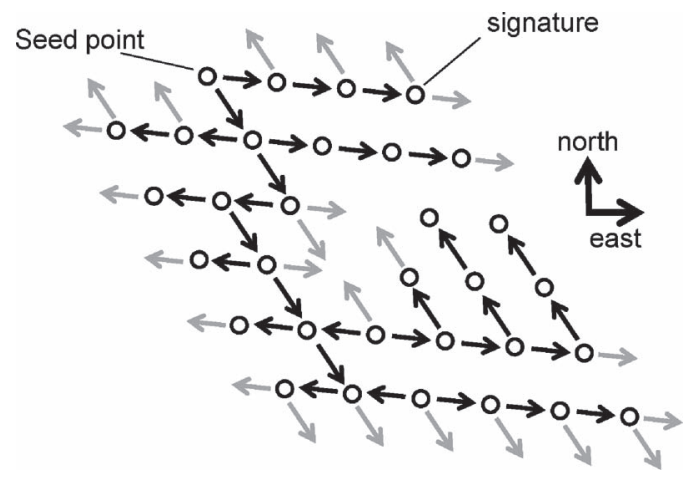

Fig. 7. Example for grouping procedure. Rows correspond to regularities in range, e.g., vertically arranged facade structures. Columns represent the arrangement of signatures along the facade, e.g., structures on the same floor level.

and column coordinates. To avoid this, a consistency check is made, analyzing every topology matrix entry for its topological correctness. As a byproduct, the consistency check enables the ability to discard inappropriate members with little binding to the pattern. For each pattern member, the direction angle $\alpha_{\text {sig }}$ and the subpixel distance $d_{\text {sig }}$ are calculated with respect to all neighbors in the 2-D pattern. For a positive vote, the difference between $\alpha_{\text {sig }}$ and the expected direction angle-given by the spacing values $\Delta x_{\text {row }}, \Delta r_{\text {row }}, \Delta x_{\text {col }}$, and $\Delta r_{\text {col }}$-has to be smaller than

$$
\Delta \alpha_{\text {row }}=\operatorname{atan}\left(\frac{d_{r}}{\sqrt{\Delta x_{\text {row }}^{2}+\Delta r_{\text {row }}^{2}}}\right)
$$

along the facade and

$$
\Delta \alpha_{\mathrm{col}}=\operatorname{atan}\left(\frac{d_{r}}{\sqrt{\Delta x_{\mathrm{col}}^{2}+\Delta r_{\mathrm{col}}^{2}}}\right)
$$

in range (quadrants to be considered). In addition, the difference between $d_{\text {sig }}$ and the expected spacing has to be smaller than $d_{r}$, respectively.

As a result, up to four votes are obtained for each pattern member. A topology matrix entry is kept if the number of positive votes is greater than or equal to the number of negative votes (avoid topological error) or if only one vote is obtained (avoid isolated members). Afterward, rows and columns at the pattern margin are removed if they have less than $\min _{r}$ and $\min _{c}$ entries (iterative top-down, bottom-up, left-right, and right-left). The cropping is continued until the topology matrix remains stable. Finally, the resulting topology matrix is accepted if it fulfills the predefined size criterion (minimum number of rows $\min _{r}$ and columns $\min _{c}$ ). As a counterpart to the flexible grouping procedure, the consistency check and cropping serve the structure and compactness of the resulting pattern.

\section{E. Iterative Analysis}

The pattern recognition algorithm has been implemented in an iterative manner in order to analyze the set of remaining pattern candidates for additional patterns. To this end, a new layover image is created from the original one by discarding local windows containing all already selected pattern members. The next iteration is started with the analysis of the Hough space, where the binary image is created from the remaining pattern candidates. The updated layover image is then cropped based on the line fitting result before conducting the spectral analysis. So far, the iterative procedure has been tested with a variety of simulated patterns. Appropriate layover examples from TerraSAR-X images have not been identified/tested so far.

\section{CASE STUdies}

Two case studies are presented in this paper, which have been selected based on the availability of high-resolution spotlight TerraSAR-X images and DSMs (simulation step) as well as on the presence of prominent facade grammars/signature patterns. Moreover, the case studies exemplify the impact of parameter selection, changing perspectives, intensity variations, and layover interferences. Finally, the results serve the discussion of the use of topological information in the context of PSI, radargrammetry, and change detection.

The parameters related to the geocoded SAR data are included as constants into the processing chain (spatial resolution: $1.1 \mathrm{~m}$ in azimuth and $0.6 \mathrm{~m}$ in range; pixel spacing: $0.5 \mathrm{~m}$ in east and north). The uncertainty of the rotation angles may rely on the geocoding procedure and the layover extraction step. For the case studies, values are selected based on our experience. The geocoding angle is expected to be known precisely $\left(\Delta_{\theta r}= \pm 1^{\circ}\right)$, whereas the facade orientation is related to more uncertainty $\left(\Delta_{\theta s}= \pm 3^{\circ}\right)$.

\section{A. Facade "Nordbau TUM"}

The first case study aims at the extraction of patterns for different imaging perspectives. The facade of interest is located at the campus of Technische Universität München (TUM). Fig. 8 shows a perspective view on the facade. Regularly distributed metallic window frames on six floors are part of a stepped facade surface made of bricks. The two geocoded TerraSAR-X layover images to be analyzed are shown in Figs. 9(a) and 10(a). The images correspond to incidence angles of $50.0^{\circ}$ (perspective 1) and $39.1^{\circ}$ (perspective 2) and have been captured from a descending orbit. Black pixels represent the area on the outside of the simulated layover mask. The image pixels cannot be compared directly as the layover distortion effect is different. However, both layover areas contain signature regularities likely related to the regular arrangement of windows on the facade. Therefore, a comparison of the layover patterns is pursued.

The parameters for pattern extraction are kept constant for both images, i.e., the minimum pattern size $\left(\min _{r}=3, \min _{c}=\right.$ 3 ), the factor for extracting candidates in the Hough space $\left(f_{H}=0.3\right)$, and the distance criteria $\left(d_{i}=1.5\right.$ pixels, $d_{r}=$ 1.5 pixels). First, pattern candidates are selected based on radiometric constraints (local maxima and similarity of intensities; cf., Section III-A). The result for perspective 1 is shown in Fig. 9(b). After extracting lines in the Hough space, the 


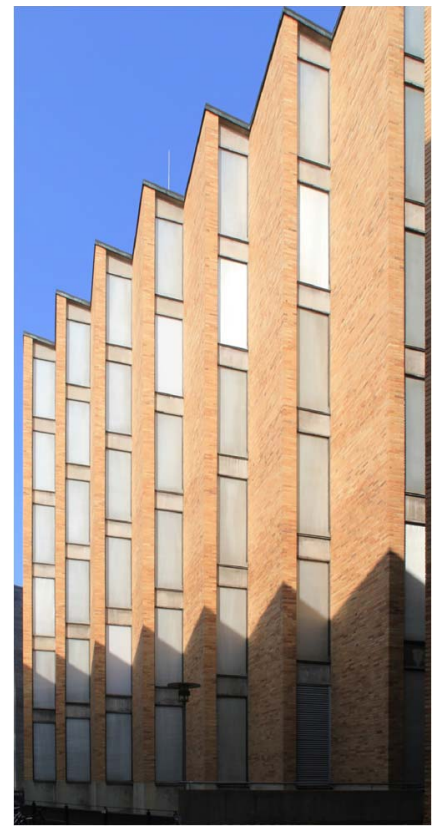

Fig. 8. Facade "Nordbau TUM" with six floors and seven windows each.

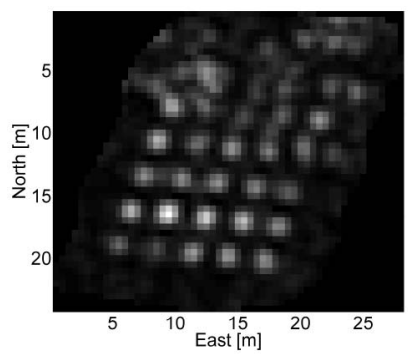

(a) Layover image ( $48 \times 56$ pixels)

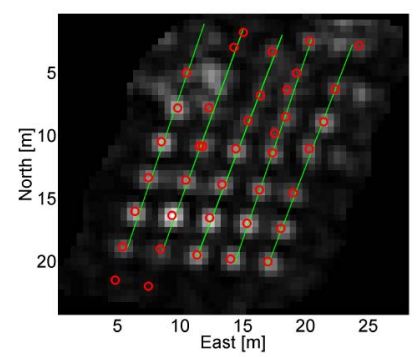

(c) Signatures fitting to lines (38)

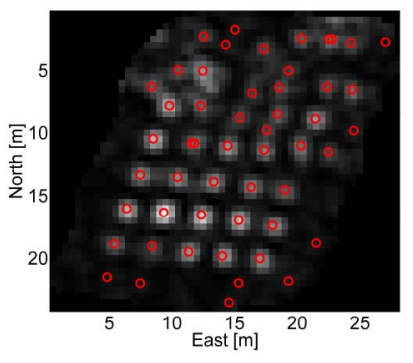

(b) Pattern candidates (52)

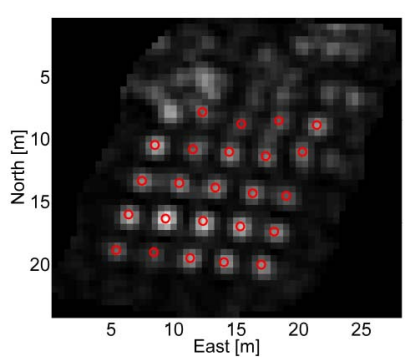

(d) Extracted pattern
Fig. 9. Extracted pattern of facade "Nordbau TUM" for incidence angle 50.0 . Parameters: $\min _{r}=3, \min _{c}=3, d_{i}=1.5$ (pixels), $d_{r}=$ 1.5 (pixels), and $f_{H}=0.3$. Note that analysis is based on the full range of intensities, whereas the visualized SAR images are clipped (8-b gray values).

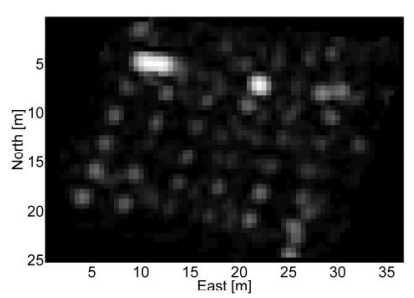

(a) Layover image ( $50 \times 73$ pixels)

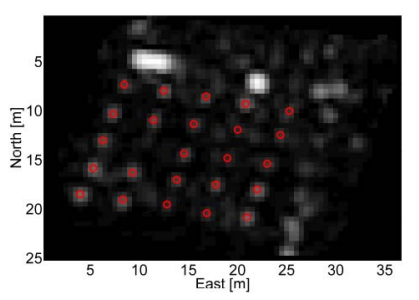

(b) Extracted pattern
Fig. 10. Extracted pattern of facade "Nordbau TUM" for incidence angle $39.1^{\circ}$. The parameters for pattern extraction are the same as those for the result shown in Fig. 9.

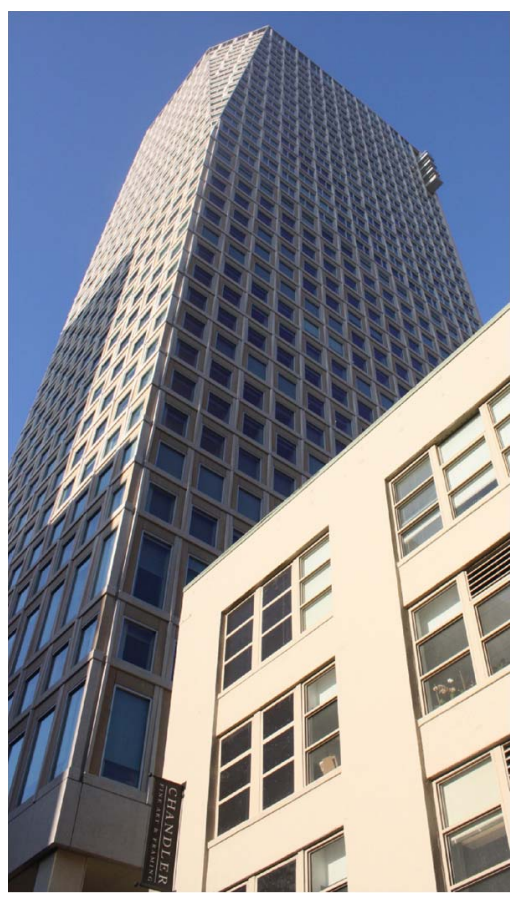

Fig. 11. Skyscraper facade in San Francisco city center. Different window frames are present on the upper and lower parts of the facade (lower part: 15 floors with 15 windows; upper part: 17 floors with window number decreasing from 15 to 11). Moritz Rexer, TUM.

pattern candidates are matched to the lines (cf., Section III-B), decreasing the number of pattern candidates (see Fig. 3 for perspective 1; note that the lines cover the full layover area, whereas only the overlapping area is visualized in the figure). The spectral analysis is conducted based on local windows extracted at the remaining pattern candidates in order to emphasize dominant frequencies (see Fig. 4). The extracted spectrum peaks for the facade "Nordbau," containing the spacing information for the grouping step, are marked in Fig. 6. Note that only a small part of the spectrum is used for identifying the peaks (cf., Section III-C).

After the grouping procedure and the inherent consistency check, the final pattern matrices are obtained for both perspectives (visualized in Figs. 9(d) and 10(b); cf., Section III-D). For perspective 1, the topology matrix contains five columns with five signatures (including one zero marking a pattern position without an appropriate member). Four signatures have been discarded by the consistency check, and four signatures have been discarded by matrix cropping. For perspective 2, the topology matrix contains five columns with five signatures including one zero. Four signatures have been discarded by the consistency check, and three signatures have been discarded by matrix cropping. In comparison to seven columns in reality (cf., Fig. 8), the result indicates that, for both perspectives, two vertical columns of window corners are occluded and hence do not backscatter the radar signal. Nonetheless, the detected patterns allow the ability to confirm layover regularities and, hence, the presence of the facade in both images. The computation times of the whole algorithm are $0.09 \mathrm{~s}$ for perspective 1 and $0.15 \mathrm{~s}$ for perspective 2 (MATLAB code, working station: Dual Core 3.17 GHz, Intel Core 2 Duo). 


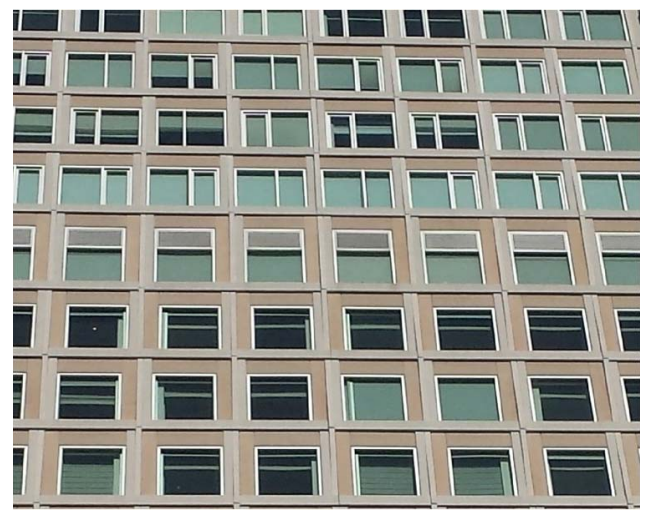

Fig. 12. Close-up view of the transition zone between the lower facade part (uniform spacing between window corners) and upper part (two corners per window frame). Cameron Bingley, San Francisco State University.

\section{B. Facade "Skyscraper," San Francisco}

The second case study deals with a large layover area of a skyscraper (height: $147 \mathrm{~m}$ ). Fig. 11 shows a perspective view of the building of interest which is located in the center of San Francisco. The lower part is covered by square windows with regular spacing in between (15 floors with 15 windows). In contrast, the facade grammar is different on the upper part, where the rectangular window frames contain two glass surfaces of different sizes (17 floors with the number of windows decreasing from 15 to 11). The window frames are made of metallic material, whereas the surrounding surfaces are covered by plain wall tiles or plaster with moderate roughness. Fig. 12 shows the transition zone between the lower and upper parts of the facade. It can be seen that:

1) on both facade parts, the spacing between windows repeats on every second floor level (small shifts also on the lower part of the facade);

2) on the upper facade part, the composition of glass surfaces repeats on every second floor level;

3) the facade is characterized by trihedrals with little roughness where point-like signatures are expected to dominate.

The layover image is shown in Fig. 13(a), corresponding to the upper half of the red layover outline in Fig. 2). It has been captured by TerraSAR-X in high-resolution spotlight mode from a descending orbit with an incidence angle of $39.3^{\circ}$. The layover contains irregular parts with high intensities (isolated point signatures and linear patches of signatures), whereas regular parts are only represented by moderate intensity. The regularity on the upper facade half is disturbed due to the changed window type (two corners per window, changing arrangement of glass surfaces on adjacent floors). Further disturbances are likely due to coinciding layover areas of adjacent buildings (see Fig. 2).

For extracting the pattern, the parameters $\min _{r}=3, \min _{c}=$ $3, d_{i}=2$ (pixels), and $f_{H}=0.2$ are kept constant. In contrast, the distance threshold $d_{r}$ for the grouping shows strong relevance on the result. In the following, two solutions are compared based on $d_{r}=2$ (pixels; solution 1) and 1 (pixel; solution 2). The computation time is $1.32 \mathrm{~s}$ for each solution. Fig. 13(b) shows the selection of pattern candidates that fulfill

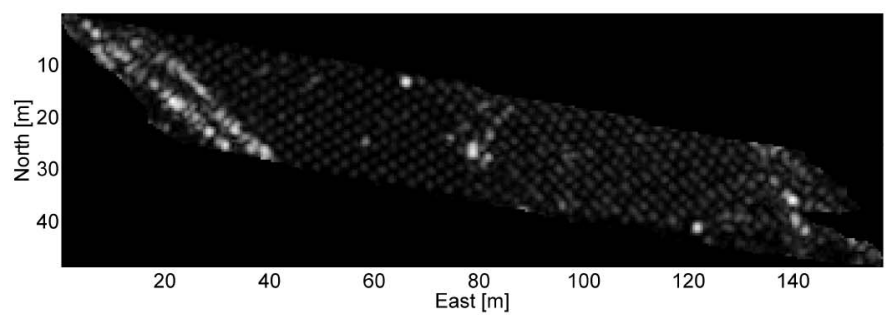

(a) Layover image (97 x 314 pixels)

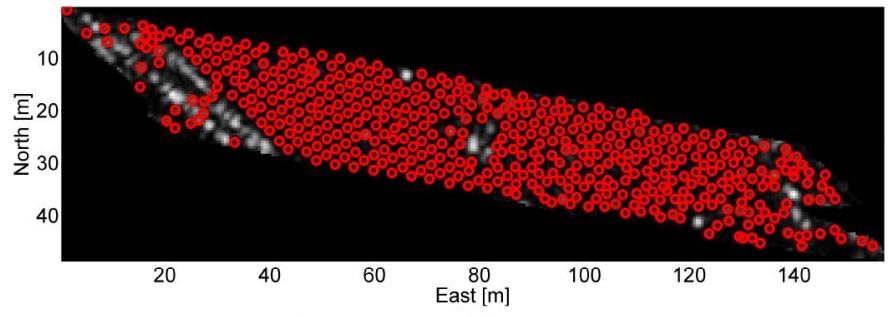

(b) Pattern candidates (449)

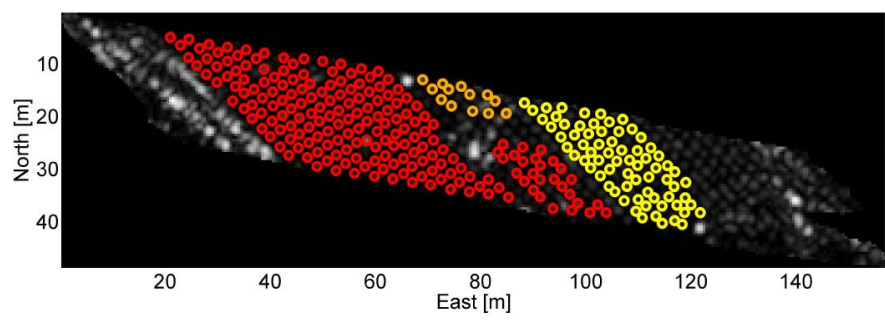

(c) Solution 1: 4 extracted patterns based on distance criterion $d_{r}=2$ (pixels)

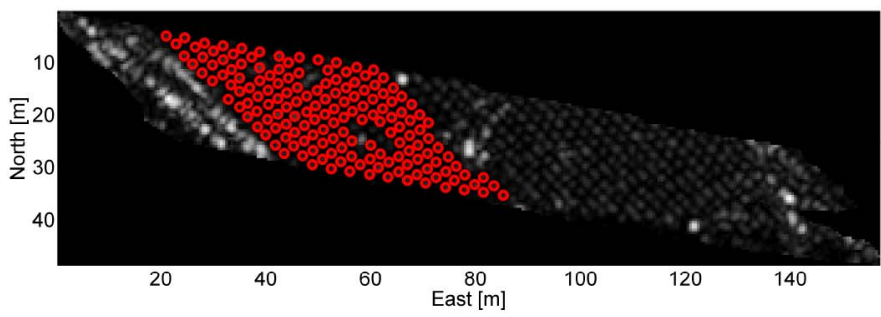

(d) Solution 2: extracted pattern based on distance criterion $d_{r}=1$ (pixels)

Fig. 13. Pattern extraction for facade "Skyscraper" (incidence angle: $39.3^{\circ}$ ). Constant parameters: $\min _{r}=3, \min _{c}=3, d_{i}=2$ (pixels), $f_{H}=0.3$, and pixel spacing: $0.5 \mathrm{~m} \times 0.5 \mathrm{~m}$. Spatial resolution: $0.6 \mathrm{~m}$ (range) and $\times 1.1 \mathrm{~m}$ (azimuth). Clipped SAR image visualized in 8-b gray values.

the intensity criteria. It can be seen that the majority of irregular signatures are discarded. Moreover, it has to be noted that the facade grammar at the upper facade half-presumably two corner reflectors per window $=30$ in total—is not present in the TerraSAR-X image. Hence, problems are expected in extracting the pattern for that part of the layover.

Based on the spacing values from the spectral analysis, the pattern within the layover area is extracted by the grouping procedure. Solution 1 is visualized in Fig. 13(c), consisting of one pattern covering the lower facade half (12 columns with 15 signatures each, 12 zeros) and three patterns representing the upper facade half (6 columns with 15 signatures each, 19 zeros; 8 columns with 4 signatures each, 5 zeros; 4 columns with 4 signatures each, 3 zeros). Ten candidates have been discarded by the consistency check, and seven candidates have been discarded by matrix cropping. Solution 2 , whose pattern 
is shown in Fig. 13(d), is based on stronger restrictions on the grouping and, thus, is more sensible to the regularity at the facade. After discarding one candidate based on consistency without the need for matrix cropping afterward, a pattern of the size 12 columns times 15 rows is obtained (18 zero entries).

Solution 2 resembles reality more closely as it indicates that only the lower part of the facade is characterized by a uniform spacing between the reflecting window corners (neglecting small relative shift of windows). However, solution 1 better preserves the overall regularity. Even if the true grammar at the upper facade part is generalized to a simplified and uniform one in the final topology matrix, more building hints are provided for a larger part of the layover.

\section{Discussion}

Both case studies confirm the relevance of the methodological components contained in the algorithm. The radiometric analysis is crucial for selecting the initial set of pattern candidates. Fitting the candidates to lines extracted from the Hough space reduces the number of outliers (update of pattern candidates) and supports the subsequent spectral analysis. Finally, 2-D patterns are extracted from the updated pattern candidates by a directed grouping procedure supported by a consistency check.

At the present state of the method, six parameters have to be selected manually, whereof two are crucial for the resulting patterns. On the one hand, the distance criterion $d_{i}$ is important to discard spatial outliers from the set of pattern candidates. It should not be selected too strictly (e.g., 1.5-3 pixels for TerraSAR-X spotlight mode) as actual pattern members may be mistakenly discarded. Without the criterion, the linear arrangement of members in the final pattern would reveal strong disturbances. On the other hand, the distance criterion $d_{r}$ steers the grouping procedure when extracting the pattern. The selection of the parameter should be based on the subsequent use of the extracted patterns. As an example, the criterion should be strict when grouping PS in a stack (less than 1.5 pixels in TerraSAR-X spotlight mode) and moderate for detecting changes in two SAR images (e.g., 2-3 pixels for TerraSAR-X spotlight mode). Different values have been tested for the distance criteria, reaching up to $d_{i}=3$ (pixels) and $d_{r}=$ 3 (pixels) for identifying patterns in heavily disturbed layover areas.

The selection of the distance criteria $d_{i}$ and $d_{r}$ is empirical so far. As a future improvement, the parameters may also rely on an intelligent selection based on the application (requirements in the context of change detection, PSI, or radargrammetry) or the image content (regularity, number, and appearance of signatures in the facade layover area).

Four parameters are kept constant for the case studies presented in this paper. The minimum number of rows and columns of patterns is only used to avoid small subpatterns. Alternatively, prior knowledge about the building dimensions or building type may be considered for further restricting the pattern extraction. The maximum spacing value $d_{s}$ (meters) between facade elements is constant and a realistic choice for most buildings. Finally, the Hough space truncation factor $f_{H}$ enables us to identify line candidates in the Hough space. For TerraSAR-X spotlight images, a value of 0.3 has proven to be a reasonable compromise. Especially for noisy layover areas, lower values should be avoided as too many candidates remain for the grouping step, resulting in fake patterns without correspondence to reality.

Altogether, the potentials of the algorithm can be summarized as follows.

1) The relation of signatures in facade layovers in single SAR images can be characterized for different intensity levels and apparent layover disturbances.

2) The algorithm is sensible to uniform spacing of repeating facade structures but also indicates potentials on extracting pseudoregularities for complicated facade grammar, which could be used as building hints (e.g., for representing facade grammar not spatially resolved by the SAR sensor).

3) The spectral analysis provides spacing values dominating the facade layover, avoiding local tests in advance to the grouping procedure.

4) The grouping procedure allows us to characterize spatially separated layover regularities as no assumption is made on the number of patterns. Moreover, the combination of grouping and consistency check favors reliable solutions as the signatures in the pattern have to be connected.

On the contrary, the case studies also reveal limitations to be discussed. For instance, the separation of real regularities from pseudoregularities is not tackled so far. The decision may be supported by the parameter selection [e.g., minimum matrix size in Fig. 13(d)] but also by an additional evaluation step after the grouping procedure. The proposed method can be applied for analyzing disturbed layover areas as exemplified in Fig. 14. Two subpatterns are extracted, which likely correspond to facade structures distributed over three building floors. On the one hand, the patterns confirm the presence of facade structures as a building hint. On the other hand, knowledge about the floor level could be exploited in PSI processing. To this end, however, connecting the subpatterns beforehand would be desirable.

The proposed algorithm relies on the presence of point signature regularities in layover areas. This has to be considered when integrating the algorithm into SAR applications. Given the signature regularities in the scene, the extracted patterns can be used for object identification/characterization and for comparing images captured from different perspectives [change detection and radargrammetry; e.g., see results in Figs. 9(d) and 10(b)]. In this context, object occlusions depending on the imaging geometry have to be taken into account. Relaxing the pattern constraints may be welcomed in the context of change detection, e.g., for confirming whether building parts are still present or not [see Fig. 13(c) as an example]. Also, radargrammetric methods may benefit from the extended amount of layover information when relating pairs of signatures in SAR images captured from different perspectives. In contrast, the grouping of PS at facades may rely on more constrained solutions as shown in Fig. 13(d). 


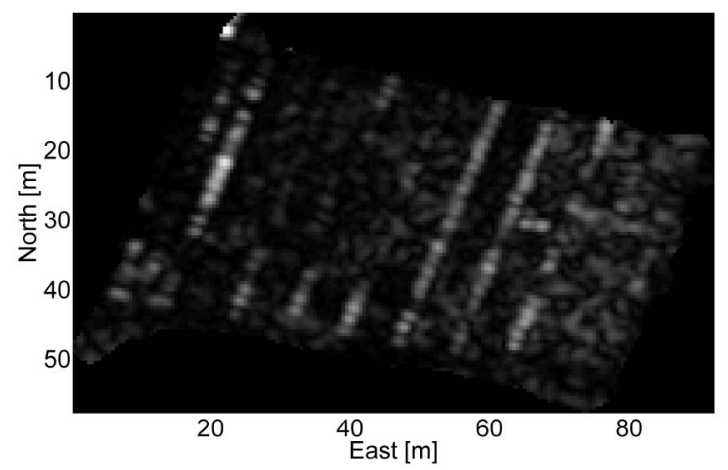

(a) Layover image

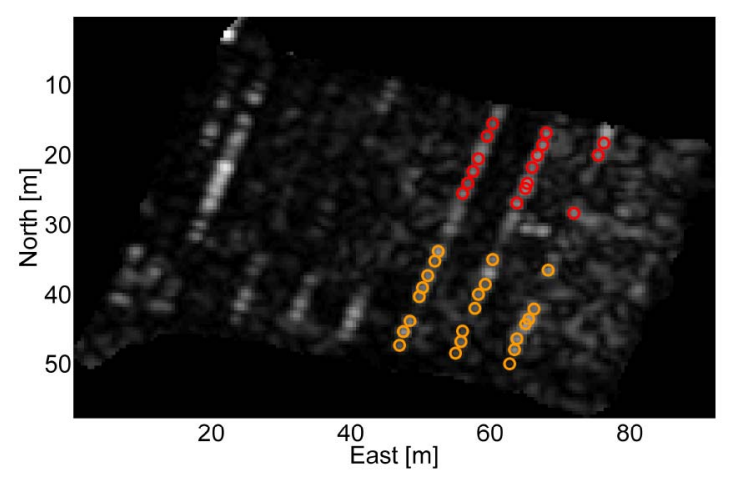

(b) Extracted pattern

Fig. 14. Extracted pattern for disturbed layover (facade in Munich city center). Parameters: $\min _{r}=3, \min _{c}=3, d_{i}=1.5$ (pixels), $d_{r}=3$ (pixels), and $f_{H}=0.3$.

\section{Conclusion And Outlook}

Regular arrangements of facade structures such as window or balcony corners are often related to signature regularities in the respective layover area in high-resolution SAR images. By exploiting this information, this paper has presented a new algorithm for extracting and characterizing patterns of pointlike signatures in single SAR images. The method follows the assumption of intensity similarity, linearity, and regularity. It is formulated on a general basis, making the method applicable to SAR images in azimuth/range geometry and to geocoded data. Two case studies on geocoded data (facade of TUM and facade of a skyscraper in San Francisco) have been presented in detail to indicate the potentials and limitations for applications such as PSI, radargrammetry, and change detection. Regular signature arrangements can be identified, even for low intensity levels. Extracted pseudopatterns related to facade grammar not resolved by the SAR sensor may be used as building hints. Future work may be concerned with realizing the interface to PSI and radargrammetric methods in order to make use of extracted patterns. In this context, data-driven or applicationdriven strategies will be necessary for supporting the parameter selection.

\section{ACKNOWLEDGMENT}

The authors would like to thank Airbus Defence and Space for providing the TerraSAR-X data set of San Francisco (IEEE Data Fusion Contest 2012) and C. Bingley, San Francisco State
University, and M. Rexer, TU München, for providing the photographs of the skyscraper facade.

\section{REFERENCES}

[1] U. Soergel, U. Thoennessen, A. Brenner, and U. Stilla, "High-resolution SAR data: New opportunities and challenges for the analysis of urban areas," Proc. Inst. Elect. Eng.-Radar, Sonar Navigat., vol. 153, no. 3, pp. 294-300, Jun. 2006.

[2] U. Stilla, "High resolution radar imaging of urban areas," in Proc. Photogramm. Week, 2007, pp. 149-158.

[3] R. Guida, A. Iodice, D. Riccio, and U. Stilla, "Model-based interpretation of high-resolution SAR images of buildings," IEEE J. Sel. Top. Appl. Earth Observ., vol. 1, no. 2, pp. 107-119, Jun. 2008.

[4] S. Auer, S. Gernhardt, and R. Bamler, "Investigations on the nature of persistent scatterers based on simulation methods," in Proc. JURSE, 2011, pp. 61-64.

[5] S. Gernhardt and R. Bamler, "Deformation monitoring of single buildings using meter-resolution SAR data in PSI," ISPRS J. Photogramm. Remote Sens., vol. 73, pp. 68-79, Sep. 2012.

[6] X. Zhu and R. Bamler, "Very high resolution spaceborne SAR tomography in urban environment," IEEE Trans. Geosci. Remote Sens., vol. 48, no. 12 , pp. 4296-4308, Dec. 2010.

[7] A. Ferro, D. Brunner, and L. Bruzzone, "Automatic detection and reconstruction of building radar footprints from single VHR SAR images," IEEE Trans. Geosci. Remote Sens., vol. 51, no. 2, pp. 935-952, Feb. 2013.

[8] U. Soergel, E. Michaelsen, A. Thiele, E. Cadario, and U. Thoennessen, "Stereo analysis of high-resolution SAR images for building height estimation in cases of orthogonal aspect directions," ISPRS J. Photogramm. Remote Sens., vol. 64, no. 5, pp. 490-500, Sep. 2009.

[9] E. Simonetto, H. Oriot, and R. Garello, "Rectangular building extraction from stereoscopic airborne radar images," IEEE Trans. Geosci. Remote Sens., vol. 43, no. 10, pp. 2386-2395, Oct. 2005.

[10] W. Pitz and D. Miller, "The TerraSAR-X satellite," IEEE Trans. Geosci. Remote Sens., vol. 48, no. 2, pp. 615-622, Feb. 2010.

[11] S. Gernhardt, "High Precision 3D Localization and Motion Analysis of Persistent Scatterers Using Meter-Resolution Radar Satellite Data," Ph.D. dissertation, Deutsche Geodätische Kommission, Verlag der Bayerischen Akademie der Wissenschaften, München, Germany, Reihe C, Nr. 672, 2012. [Online]. Available: http://dgk.badw.de/fileadmin/docs/ c-672.pdf

[12] E. Michaelsen, U. Soergel, and U. Stilla, "Grouping salient scatterers in InSAR data for recognition of industrial buildings," in Proc. 16th Int. Conf. Pattern Recognit., 2002, vol. 2, pp. 613-616.

[13] E. Michaelsen, U. Soergel, and U. Thoennessen, "Perceptual grouping for automatic detection of man-made structures in high-resolution SAR data," Pattern Recognit. Lett., vol. 27, no. 4, pp. 218-225, Mar. 2006.

[14] A. Schunert and U. Soergel, "Grouping of persistent scatterers in highresolution SAR data of urban scenes," ISPRS J. Photogramm. Remote Sens., vol. 73, pp. 80-88, Sep. 2012.

[15] L. Schack, A. Schunert, and U. Soergel, "Lattice detection in persistent scatterer point clouds and oblique aerial imagery," in Proc. IGARSS, 2012, pp. 451-454.

[16] S. Auer, S. Gernhardt, and R. Bamler, "Ghost persistent scatterers related to multiple signal reflections," IEEE Geosci. Remote Sens. Lett., vol. 8, no. 5, pp. 919-923, Sep. 2011.

[17] S. Gernhardt, S. Auer, and K. Eder, "Persistent scatterers at building facades-Evaluation of appearance and localization accuracy," ISPRS J. Photogramm. Remote Sens., 2014, online available.

[18] A. Thiele, M. Wurth, M. Even, and S. Hinz, "Extraction of building shape from TanDEM-X data," in Proc. Int. Archives Photogramm., Remote Sens. Spatial Inf. Sci., 2013, vol. XL-1/W1, pp. 345-350.

[19] S. Auer, "3D Synthetic Aperture Radar Simulation for Interpreting Complex Urban Reflection Scenarios," Ph.D. dissertation, Deutsche Geodätische Kommission, Verlag der Bayerischen Akademie der Wissenschaften, München, Germany, Reihe C, Nr. 660, 126 p., 2011. [Online]. Available: http://dgk.badw.de/fileadmin/docs/c-660.pdf

[20] J. Tao, S. Auer, G. Palubinskas, P. Reinartz, and R. Bamler, "Automatic SAR simulation technique for object identification in complex urban scenarios," IEEE J. Sel. Topics Appl. Earth Observ., vol. 7, no. 3, pp. 9941003, Mar. 2014.

[21] J. Tao, S. Auer, P. Reinartz, and R. Bamler, "Object-based change detection for individual buildings in SAR images captured with different incidence angles," in Proc. IGARSS, 2013, pp. 1238-1241. 
[22] J. Groot and M. Otten, "SAR imaging of corner reflectors larger than the spatial resolution," IEEE Trans. Geosci. Remote Sens., vol. 32, no. 3, pp. 721-724, May 1994.

[23] C. Oliver and S. Quegan, Understanding Synthetic Aperture Images. Johnson City, NY, USA: SciTech Publ., Inc., 2004.

[24] P. V. C. Hough, "Method and Means for Recognizing Complex Patterns," U.S. Patent 3069654 A, Dec. 18, 1962.

[25] R. O. Duda and P. E. Hart, "Use of the Hough transformation to detect lines and curves in pictures," Commun. ACM, vol. 15, no. 1, pp. 11-15, Jan. 1972.

[26] P. Volet and M. Kunt, "A new method for the synthesis and efficient coding of natural structured textures," in Proc. SPIE, 1986, vol. 0594, pp. 225-232.

[27] T.-I. Hsu and R. Wilson, "A two-component model of texture for analysis and synthesis," IEEE Trans. Image Process., vol. 7, no. 10, pp. 14661476, Oct. 1998

[28] F. Galasso and J. Lasenby, "Shape from texture of developable surfaces via Fourier analysis," in Proc. Int. Symp. Vis. Comput., 2007, pp. 702-713.

[29] J. G. Daugman, "Uncertainty relation for resolution in space, spatial frequency, and orientation optimized by two-dimensional visual cortical filters," J. Opt. Soc. Amer. A, vol. 2, no. 7, pp. 1160-1169, Jul. 1985.

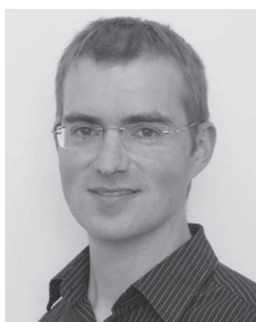

Stefan Auer received the Dipl.-Ing.(Univ.) degree in geodesy and the Dr.-Ing. degree from Technische Universität München (TUM), München, Germany, in 2005 and 2011, respectively.

$\mathrm{He}$ is a full-time Scientific Collaborator with the Chair of Remote Sensing Technology, TUM, where he is concerned with the simulation and analysis of radar signal reflections at urban structures. In the course of his doctoral dissertation, he spent three months as a Guest Researcher with the Department of Electronic and Telecommunication Engineering (DIET), University of Naples "Federico II," Naples, Italy. His main interests are the visual and automated interpretation of high-resolution synthetic aperture radar (SAR) imagery and the evaluation of the 3-D localization capability of interferometric SAR algorithms. In this context, he developed the 3-D SAR simulator RaySAR which enables the investigation of the nature of prominent SAR image signatures related to man-made structures. Based on the knowledge from simulation case studies, he is working on algorithms for characterizing signature patterns related to building facades.

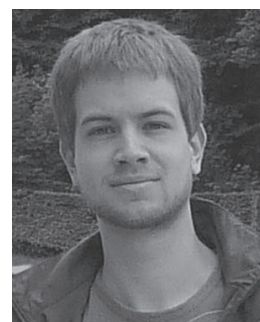

Christoph Gisinger received the Diploma degree in geodesy from Technische Universität Graz, Graz, Austria, and the M.Sc. degree in Earth oriented space science and technologies (ESPACE) from Technische Universität München (TUM), München, Germany, where he has been working toward the Ph.D. degree in joint processing of synthetic aperture radar (SAR) and global navigation satellite system to retrieve geophysical signals since March 2013

$\mathrm{He}$ is currently with the Institute for Astronomical and Physical Geodesy (IAPG), TUM. His research includes stereo SAR, correction modeling for absolute SAR observations, an radargrammetric image processing with focus on fusing SAR with geodetic observations to measure deformations.

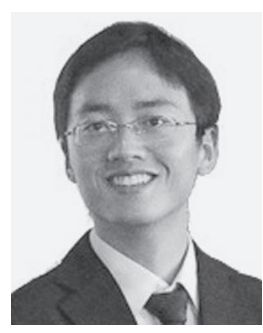

Junyi Tao was born in Wuhan, China, in 1983. He received the Dipl.-Ing.(Univ.) degree in geodesy and geoinformatics from Universität Stuttgart, Stuttgart, Germany, in 2009.

Since October 2009, he has been a Scientific Collaborator with the Remote Sensing Technology Institute (IMF), German Aerospace Center (DLR), Oberpfaffenhofen, Germany, working in close cooperation with the Chair of Remote Sensing Technology (LMF), Technische Universität München (TUM), München, Germany. In June 2012, he was a Guest Scientist with the Remote Sensing Laboratory, Department of Information Engineering and Computer Science, University of Trento, Trento, Italy. His research interests include synthetic aperture radar (SAR) simulation, SAR image interpretation, multimodal data fusion, and change detection. In particular, he focuses on the combination of LiDAR and SAR data with simulation techniques for object identification and change detection in urban areas.

Mr. Tao was the recipient of the second prize in the IEEE GRSS Data Fusion Contest in 2012, supported by his colleagues from TUM/DLR. 